www.ocula.it • ISSN 1724-7810 • Vol 21, No 23 (July 2020) • DOI: 10.12977/ocula2020-30

Fiori dell'anima. La simbologia dei fiori nell'immaginario religioso Flowers of the soul. The symbolism offlowers in the religious imagination a cura di Marco Papasidero e Francesco Galofaro

\title{
«Poma di paradiso» \\ Le descrizioni botaniche nei diari di pellegrinaggio del corpus fiorentino tra XIII e xV secolo
}

\section{Ilaria Sabbatini}

SISMEL (Società Internazionale per lo Studio del Medioevo Latino), IT

ilaria.sabbatini@sismelfirenze.it

\begin{abstract}
In the Florentine diaries of pilgrimages to the Holy Land, dated between the XIII ${ }^{\text {th }}$ and $\mathrm{XV}^{\text {th }}$ centuries, nature is always ascribed a powerfully symbolic value. This has both a metaphorical dimension and a taxonomic approach that is derived from the influence of practical writings, such as libri di famiglia. The plant species described by the Florentine diarists, even within this new naturalistic paradigm, continue to maintain the sense of wonder and miracle as if they were a tangible expression of a divine presence that animates places and nature.
\end{abstract}

\section{Keywords}

Banana; Balm; Pilgrimage journals; Pilgrims

\section{Sommario/Content}

1. I pellegrini scrittori fiorentini

2. La descrizione della natura tra deserto e giardino

3. L'oasi del balsamo

4. La banana paradisiaca

5. Conclusioni

Bibliografia 


\section{I pellegrini scrittori fiorentini}

Intorno alla metà del xiv secolo, il frate minore Niccolò da Poggibonsi si incamminava alla volta della Terra Santa per svolgere quel santo viaggio che tanti pellegrini prima di lui avevano compiuto. Per tenere memoria e rendere testimonianza dell'esperienza, il frate aveva stilato un diario in cui raccontava le cerch $^{1}$ effettuate sui Luoghi Santi, i fatti salienti del viaggio, le cose notevoli e gli eventi interessanti a cui aveva assistito. Come lui, molti altri viaggiatori avevano scritto i resoconti dei loro pellegrinaggi intrattenendosi in descrizioni di vario genere che riguardavano le popolazioni, i paesaggi antropizzati e gli ambienti naturali, in un continuum narrativo di raro interesse storico e antropologico. ${ }^{2}$ In queste descrizioni un certo spazio è spesso dedicato alle spiegazioni botaniche che non osservano, ovviamente, il rigore scientifico di un'operazione tassonomica ma rivelano comunque un grande e condiviso interesse dei viaggiatori per le piante in tutte le loro varietà. La lettura della natura presente in questi testi, che costituiscono un vero e proprio corpus dal particolare valore documentario, è indubbiamente condizionata dalla cultura di appartenenza degli autori e rivela una modalità di rappresentazione costantemente in equilibrio tra la descrizione naturalistica e la significazione religiosa. Quando Niccolò da Poggibonsi si trova di fronte al banano sente il bisogno di renderne conto ai suoi lettori e imbastisce una descrizione in cui i frutti squisiti dell'albero, fin dal loro nome, sono trasposti in una dimensione idealizzata e simbolica condensata nell'appellativo di «poma di Paradiso. ${ }^{3}$

Niccolò da Poggibonsi non è l'unico a narrare, in quel contesto e in quell'età, l'incontro con l'alterità del Vicino Oriente. Esiste infatti un vero e proprio corpus di diari toscano-fiorentini dedicati ai viaggi dei pellegrini in Terrasanta scritti tra il xiI e il xv secolo. ${ }^{4} \mathrm{Si}$ tratta di un caso particolarmente fortunato poiché non è comune, nell'odeporica italiana, rintracciare un così nutrito gruppo di testi tutti afferenti a un medesimo ambito geografico e socioeconomico. L'alto numero di testimoni concentrati nella stessa area trova la sua ragione d'essere proprio nella strutturazione dell'ambiente culturale della Firenze del tardo medioevo. I diari di pellegrinaggio che fanno parte del corpus fiorentino sono stati circoscritti in base a dei criteri oggettivi che permettono di garantirne la continuità stilistica e tematica. Ogni singolo resoconto è preso in considerazione solo se pone i Luoghi Santi al centro concettuale di un viaggio fisico, comunque ne tratti lo svolgimento; il viaggio deve essere avvenuto realmente, non sono stati contemplati i racconti di viaggi immaginari; gli autori compresi nel corpus sono fiorentini o toscani; almeno uno dei manoscritti di ciascun diario è conservato a Firenze. ${ }^{5}$

1 Con questo nome vengono indicate le visite, le soste e le devozioni compiute in luoghi specifici legati alle memorie delle vicende bibliche e cristologiche.

2 Si vedano ad esempio Richard (1981); Oursel (1988); Sumption (1993); Oursel (1993); Graboïs (1998); Cardini (2002); Chélini e Branthomme (2004-6).

3 Niccolò da Poggibonsi (1990: 145).

4 Cardini (1982).

5 Sabbatini (2014). 


\section{Ocula ${ }^{23}$}

Vol 21, No 23 (July 2020) • DOI: 10.12977/ocula2020-30

Ilaria Sabbatini | «Poma di paradiso». Le descrizioni botaniche nei diari di pellegrinaggio del corpus fiorentino tra XIII $\mathrm{e}$ XV secolo

Il corpus così delimitato comprende 18 testi di cui 9 stilati da religiosi, 4 da mercanti cristiani, 1 da un orafo, 1 da un mercante ebreo, 1 da un musico di corte e 2 da autori anonimi. Sulla base dell'analisi testuale, in comparazione con gli altri testimoni, sembra che i diari anonimi siano anch'essi ascrivibili al gusto della borghesia mercantile. A questo punto il numero dei diari collegati all'ambiente mercantile può salire a 6 a patto di tenere conto del fatto che l'ipotesi riguarda solo lo stile dei testi. ${ }^{6}$

Tabella 1. Distribuzione sociale degli autori del corpus fiorentino con indicazione dei diari sinottici.

\begin{tabular}{|c|c|c|}
\hline 1288 & Riccoldo da Montecroce & frate domenicano \\
\hline XIII sec. ex. & Anonimo Panciatichiano & \\
\hline $1346-1350$ & Nicolò da Poggibonsi & frate francescano \\
\hline 1349 & Dolcibene de' Tori & musico \\
\hline 1384 & Giorgio Gucci & mercante \\
\hline 1384 & Lionardo Frescobaldi & mercante \\
\hline 1384 & Simone Sigoli & mercante \\
\hline XIV sec. & Anonimo, Viaggio & \\
\hline 1425 & Marco di Bartolomeo Rustici & orafo \\
\hline 1431 & Mariano da Siena & prete \\
\hline 1431 & Gaspare di Bartolomeo & prete \\
\hline 1468 & Pierantonio Buondelmonti & mercante \\
\hline 1474 & Alessandro Rinuccini & frate domenicano \\
\hline 1481 & Mešullam da Volterra & mercante \\
\hline 1489 & Michele da Figline & prete \\
\hline 1489 & Zanobi del Lavacchio & prete \\
\hline 1497 & Bonsignore di Francesco Buonsignori & prete \\
\hline $1500-1504$ & Pietro Pagolo Rucellai & frate francescano \\
\hline
\end{tabular}

\section{Ibidem.}




\section{La descrizione della natura tra deserto e giardino}

Nei diari del corpus fiorentino, il contesto generale risente dell'influenza delle scritture pratiche proprie dell'ambiente mercantile come i libri di famiglia ${ }^{7}$ ma la narrazione della natura ha spesso una valenza che risponde a logiche simboliche e metaforiche. Quando si tratta di contesti naturali, i viaggiatori fiorentini tendono a rappresentarli secondo una contrapposizione molto netta che si gioca tra i due estremi dell'oasi e del deserto, della sabbia e del giardino, della sazietà e della sete. Queste polarità non sono solo i modi della rappresentazione dell'ambiente naturale ma anche delle società umane che le popolano e che a tali ambienti corrispondono.

Secondo gli studi di Ashtor sull'economia medievale del Vicino Oriente, l'Egitto, che era stato il granaio di Roma e di Bisanzio, fino all'età dei califfati produceva ancora raccolti in eccedenza tanto da poterne esportare quantità considerevoli e il frumento coltivato in Siria era esportato ovunque. La Siria aveva anche delle floride orticolture e frutticolture grazie alle quali esportava vari tipi di frutta verso le regioni adiacenti dell'impero califfale. Tutta l'area della mezzaluna fertile era interessata dalla coltivazione di datteri. Nell'area comprendente Siria, Libano e Palestina si producevano grandi quantità di mele mentre la Cisgiordania e la Transgiordania producevano fichi uva e prugne. ${ }^{8}$ La maggior parte delle terre di Siria e Palestina erano coltivate senza ricorrere all'irrigazione mentre l'Egitto era subordinato a sistemi piuttosto complessi di canali, dighe e serbatoi. Per lo studioso sembra provato che la preesistente tradizione agricola, fatta di terrazzamenti e sistemi di protezione del suolo, abbia avuto continuità fino agli inizi del dominio musulmano ma sia andata poi perdendosi a partire dall'età degli Abbasidi. Le carenze dell'agricoltura erano compensate dalla fertilità del suolo che in alcune regioni era tale da permettere raccolti abbondanti anche senza un miglioramento delle tecniche agrarie. ${ }^{9}$

Piuttosto dell'ipotesi di una progressiva riduzione delle precipitazioni, Ashtor sembra avallare la convinzione che nella Mezzaluna fertile, dopo la conquista araba, sia iniziato un periodo di trasformazioni geologiche: molti villaggi soprattutto di montagna furono abbandonati a causa dell'andamento alluvionale dei corsi d'acqua che portava a una crescente erosione del suolo a monte e contemporaneamente alla formazione di depositi alluvionali a valle. In quest'area le intense precipitazioni dei mesi invernali agiscono sui terreni franosi spazzandoli via dai pendii. Questo costituisce un danno per l'agricoltura perché porta alla scomparsa delle sorgenti e alla spoliazione del suolo più fertile. Se nell'antichità il problema veniva risolto con la costruzione di terrazzamenti, nell'età medievale e moderna l'agricoltura subisce una progressiva decadenza proprio per l'abbandono delle tecniche di manutenzione dei ter-

7 Mordenti (1997); Cicchetti e Mordenti (1985); Branca (1986).

8 Ashtor (1982: 37-41). Cfr. Nanni (2017).

9 Ashtor (1982: 44, 46). 
Ilaria Sabbatini | «Poma di paradiso». Le descrizioni botaniche nei diari di pellegrinaggio del corpus fiorentino tra XIII $\mathrm{e}$ XV secolo

reni. ${ }^{10}$ Tali considerazioni suggeriscono l'idea che nel pieno medioevo il paesaggio dell'area del Vicino Oriente fosse meno rigoglioso rispetto all'antichità e il contrasto tra aree antropizzate e aree desertiche fosse già molto netto. Dall'analisi comparata dei testi diaristici emerge con chiarezza una ricorrente opposizione tra selvatico e domestico quale chiave di lettura dell'Oltremare. La coppia antinomica è usata, in partenza, per distinguere i pastori beduini nomadi da quelli parzialmente stanzializzati ma funziona anche per distinguere più in generale la società urbana, intesa come luogo di civiltà, da tutto ciò che è ritenuto in-civile. ${ }^{11}$ Il medesimo modello interpretativo trova riscontro anche nell'alternanza deserto/giardino entro i cui schemi viene rappresentato l'ambiente naturale dell'Oltremare. ${ }^{12}$

Fin dalla concezione agostiniana della storia, l'avvento del cristianesimo aveva emendato la natura selvatica - e quindi in-civile - di tutta la terra. La massima espressione della società degli uomini, ovvero dei cristiani, consisteva nell'edificazione della civitas cui si contrapponeva l'antisocietà di coloro che ne rimanevano fuori. ${ }^{13}$ In questo contesto il nomadismo veniva giudicato come una "condizione morale" in nome della quale chi viveva al di fuori della società urbana si collocava più vicino alla natura animale che non a quella umana. L'esilio di Caino «vagus et profugus in terra» era l'archetipo di quella lontananza dalla civitas che poneva il suo vagare sotto il segno della maledizione. ${ }^{14}$

Il modello occidentale della civitas, intesa come società degli uomini, viene adattato dai pellegrini fiorentini a paradigma interpretativo del paesaggio umano e ambientale del Vicino Oriente..$^{15}$ L'ordine, l'armonia, la fertilità appartengono alla natura antropizzata mentre il deserto è il luogo dell'arsura, dell'abbandono e dell'assenza di esseri umani. Nei racconti che fanno parte di questo corpus si nota insomma un filo conduttore che collega l'organizzazione stanziale, la società urbana e il giardino, a cui vengono contrapposti il nomadismo, il deserto e tutto ciò che non è collegato alla città. Le descrizioni naturalistiche abbondano di paesaggi colmi di tutte le delizie o all'opposto di spianate riarse prive di ogni conforto. I fiumi e le fonti, gli orti e le coltivazioni illuminano il panorama rurale di toni così lussureggianti da sembrare l'oggetto di una intenzionale ridondanza. Questi stilemi rappresentativi rivelano senz'altro una matrice ricca di echi scritturali che risulta evidente se solo si pensa a quanto siano frequenti nei profeti le lodi dell'acqua che vivifica e disseta. ${ }^{16}$

Il topos della Gerusalemme celeste traboccante di acque limpide non esclude che le descrizioni dei diaristi, per quanto enfatiche, possano rispecchiare quell'attitudine alla registrazione dei fatti che è una caratteristica pre-

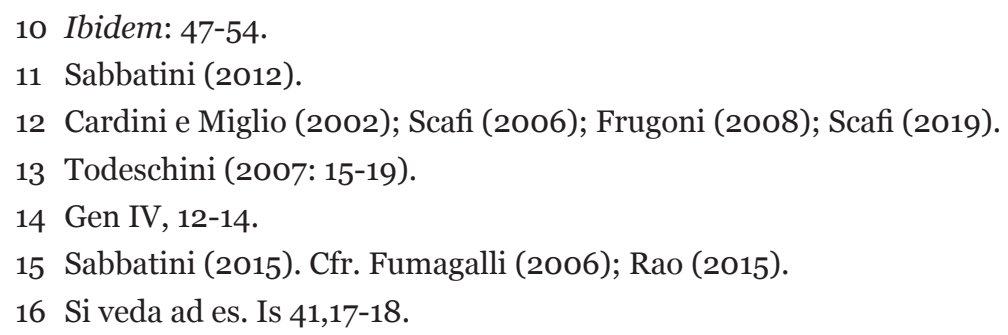




\section{Dcula ${ }^{23}$}

Vol 21, No 23 (July 2020) • DOI: 10.12977/ocula2020-30

Ilaria Sabbatini | «Poma di paradiso». Le descrizioni botaniche nei diari di pellegrinaggio del corpus fiorentino tra XIII $\mathrm{e} X \mathrm{~V}$ secolo

gnante dell'ambiente mercantile e borghese ${ }^{17}$ Protagonista nelle descrizioni naturalistiche dei pellegrini scrittori è dunque l'acqua, nell'abbondanza o nell'assenza, che nelle sue manifestazioni riesce a conferire all'ambiente l'aspetto felice e rigoglioso di un paradiso in terra. L'idea della fertilità è sempre collegata all'immaginario del fiume edenico poiché fin dal dettato scritturale la fonte che irriga il paradiso è affiancata all'albero della vita. Il testo biblico non attribuisce una virtù terapeutica intrinseca ai fiumi del paradiso ma l'idea dell'acqua è sempre associata alla fecondità fin da quando, nel Genesi, Dio separa le acque e fa germogliare la terra di ogni frutto. ${ }^{18}$ All'estremo opposto di una polarità giocata sull'equilibrio tra simbolismo religioso e rappresentazione naturalistica, il deserto rafforza negandolo il prodigio dell'acqua. In una forte descrizione del territorio desertico dell'Egitto Michele da Figline afferma: «È tutto paese sterile, sanza alcuno albore et se l'aria et la rena ardeva». ${ }^{19}$ È il Genesi stesso che riferisce i nomi dei quattro fiumi dell'Eden anche se la loro identificazione con i fiumi terrestri sarà in gran parte frutto dell'esegetica. $^{20}$

\section{L'oasi del balsamo}

Nel sistema simbolico dei pellegrini fiorentini, presso cui era presente l'idea dei fiumi paradisiaci portatori di vita, l'acqua è necessariamente accompagnata dal trionfo dell'opulenza vegetale. Dando vita a immagini di esuberante bellezza, essi si soffermano in un minuzioso elenco delle molteplici specie di piante che crescono in prossimità dei corsi d'acqua. In tutta questa fenomenologia collegata al simbolismo cristologico e alla rappresentazione edenica, un ruolo centrale viene riservato da molti autori del corpus fiorentino all'oasi di Matarea $^{21} \mathrm{e}$ alla miracolosa pianta del balsamo che in essa cresce rigogliosa ma che non può essere trapiantata altrove. La leggenda dell'oasi Matarea fa parte di quell'insieme di narrazioni, frequenti nelle scritture odeporiche, che traggono spunto dalla tradizione dei vangeli apocrifi interpolandola con i racconti popolari che circolavano tra i pellegrini probabilmente per trasmissione orale. L'oasi dove cresce il balsamo è la stessa in cui Gesù fece sgorgare un'acqua miracolosa dalle radici del sicomoro ed è soprattutto il luogo in cui, secondo il vangelo arabo dell'infanzia, si rifugiarono Maria, Giuseppe e il Bambino durante la fuga in Egitto. ${ }^{22}$

Lo storico delle religioni Craveri, a proposito dell'origine del giardino del balsamo, osserva trattarsi di una leggenda risalente al xiII secolo, frutto di un'interpolazione da parte dello stesso apocrifo che la riferisce. ${ }^{23}$ Dunque sia-

17 Cicchetti e Mordenti (1982: 1127).

18 Gen I, 9-11.

19 Michele da Figline (2010: 66).

20 Gen II, 10-14.

21 Matarea è l'attuale Matarieh, località a circa dieci chilometri dal Cairo.

22 Craveri (2002: 127).

23 Ibidem, nota 2. 


\section{Ocula ${ }^{23}$}

Vol 21, No 23 (July 2020) • DOI: 10.12977/ocula2020-30

Ilaria Sabbatini | «Poma di paradiso». Le descrizioni botaniche nei diari di pellegrinaggio del corpus fiorentino tra XIII $\mathrm{eXV}$ secolo

mo di fronte a un mito che ha avuto origine e diffusione nella fase postcrociata e probabilmente proprio a partire dai racconti dei pellegrini che venivano trasmessi anche in forma orale. L'ipotesi di Craveri è particolarmente interessante dal momento che colloca la leggenda in coincidenza con una fase di grande mutamento del genere odeporico-devozionale: quella in cui agli schemi narrativi antecedenti si assommano i toni e gli elementi dei racconti agiografici e della novellistica. ${ }^{24}$ Giorgio Gucci fornisce nel suo diario una traccia di quella che potrebbe essere la palingenesi della leggenda di Matarea: egli racconta infatti di un giardino voluto dal sultano a breve distanza dalla capitale politica. Un giardino magari costruito sfruttando una sorgente naturale, un'oasi nel deserto che proprio per il suo straordinario rigoglio nel contesto di un ambiente naturale ostile, è facilmente interpretabile come il risultato di un ineffabile intervento divino:

Prima [nel deserto del Sinai n.d.r.] trovamo una fonte molto abondante d'acqua: ed è la fonte bellissima, e così l'acqua bella e buona [...]. Ed è questa fonte posta in uno abituro fatto per lo soldano, il quale abituro secondo loro usanza è bello e grande abituro. Ed èvi il giardino del soldano grandissimo e bello, pieno di datteri e di molti buoni frutti; e in questo giardino è il luogo dove nasce il balsimo, che più in niuno altro luogo none nasce. $\mathrm{E}$ istannovi più fattori e iscrivani per lo soldano a costodia di questo giardino e a coltivallo, e li scrivani a iscrivere il balsimo che si ricoglie. Fanne il soldano grande solenità perché no'gli sia tolto. Dicesi che essa fonte nostro Signore fece coi piedi a pitizione della sua madre quando di Sorìa si fuggirono e pervennono in Egitto: perché, essendo passati per lo diserto ch'è sterile d'acqua, nostra Donna avendo sete, e non vi essendo acqua, domandando bere al suo figliuolo, esso, come è detto, col piede fece detta fonte. $\mathrm{E}$ in essa fonte nostra Donna lavò le peze del suo figliuolo e tesele nello detto orto in su albuscelli, che dapoi hanno fatto il balsimo. E queste cose sono assai verisimili: l'una per la sua bontà e potenza, l'altra perché [...] il paese è sterile d'acqua, e in tutti quelli paesi altra acqua che quella non si truova, e quella è molto abondante e perfettamente buona. E colla detta acqua inaffiano il giardino del soldano [...] né più adietro né più inanzi si fa o puote fare del detto balsimo, se none ivi nel propio luogo $\mathrm{e}$ nel cerchiòvito d'esso campo. ${ }^{25}$

Le leggende non sono soltanto quelle nobili narrazioni che celebrano fatti o personaggi fondamentali per la storia di un popolo ma possono anche consistere in più semplici racconti che, partendo da elementi reali, riescono a spiegare qualche caratteristica dell'ambiente naturale. Quella tra il giardino e il deserto è una dicotomia che si presta agevolmente a una lettura simbolica ed escatologica: il territorio arido e bruciante dell'assenza si arrende alla natura rigogliosa sbocciata intorno alla presenza del divino. Il senso profondo di questa esperienza che si trasforma in racconto ed exemplum lo si coglie nel superamento di quella cruciale linea di confine che separa il territorio ostile dal panorama familiare: «Ed ivi finisce il diserto e truovasi terreno dimestico,

24 Bartolini e Cardini (1991: 76). Cfr. Sabbatini (2019).

25 Giorgio di Guccio Gucci (1990: 271). 


\section{Dcula ${ }^{23}$}

Vol 21, No 23 (July 2020) • DOI: 10.12977/ocula2020-30

Ilaria Sabbatini | «Poma di paradiso». Le descrizioni botaniche nei diari di pellegrinaggio del corpus fiorentino tra XIII $\mathrm{e}$ XV secolo

abitato e fruttuoso. E quivi trovando dell'acqua buona ed abondantemente di buoni datteri, uve fresche, non meno che in paradiso ci parve giugnere». ${ }^{26}$

Attraverso la mediazione dei Commentaria in genesim, in cui il poeta Claudio Mario Vittore (v sec. d.C.) descrive l'Eden ricalcando i passi di Flavio Giuseppe (I sec. d.C.), si arriva agli autori del XII secolo come l'inglese Robert Pullen (XIII sec.) che nelle sue Sententiae riprenderà queste letture interpretando il Nilo come uno dei fiumi edenici. ${ }^{27}$ Anche per questa ragione l'oasi di Matarea riveste un ruolo così importante nella narrazione dei pellegrini fiorentini. Essi la descrivono secondo uno stilema idealizzato e atemporale, con evidente riferimento alla tradizionale iconografia edenica, ma al contempo la collegano con la leggenda del balsamo a completamento e integrazione dell'immagine del giardino di delizie. Sono richiami tematici che non implicano la reale convinzione di una sovrapponibilità dell'oasi cairota con il paradiso del Genesi. Ciò non di meno rappresentano echi letterari di rara potenza evocativa, capaci di stabilire una continuità tra il paradiso terrestre della creazione e la promessa escatologica dell'emancipazione dalla morte. Non è un caso infatti se al balsamo che popola il giardino sono attribuite due prerogative fondamentali: la capacità di guarire istantaneamente i corpi e l'impossibilità di essere trapiantato in un luogo diverso da quello in cui è nato. Il balsamo compie il prodigio della sua stessa esistenza e viene rappresentato spesso dai pellegrini fiorentini nella forma di un arbusto cespuglioso che, se inciso, trasuda un olio dotato di proprietà terapeutiche, come nella descrizione che ne fa Giorgio Gucci:

In questo campo fummo, e vedemo e toccamo il detto balsimo; e sagretamente noi veggenti e presenti ce ne facemo cogliere una piccolissima ampolla, che costò catuna due ducati d'oro. [...] Egli è fatto in questo modo: prima egli fa cespugli alti da terra circa a uno braccio, ed è il loro giro intorno intorno da braccio uno in uno e mezo, ch'è assai maggiore l'uno all'altro d'alteza e d'ampiezza. Poi ha la sua foglia come la mortina, ${ }^{28}$ ed ha la sua buccia come la vermena ${ }^{29}$ dello ulivo [...]. Quelli che sono usi di côrre $^{30}$ detto balsimo [...] levano questa così fatta vermene d'in sul ramo grosso ch'esce di terra [...]. E come è schiantata, comincia a gemere, ${ }^{31}$ a modo che fa la vite di marzo, e gitta a modo di cotali lagrime piccole e rade. Ed eglino con uno poco di bambagia battuta ricolgono il detto balsimo, e quando hanno piena la bambagia, la priemono nella ampolla [...]. L'udore che gitta detto balsimo, e i rami e le foglie, è inistimabile, quanto è grande, soave e confortativo; e di certo egli eccede tutti gli altri udori terreni, e sia quale cosa essere vuole. ${ }^{32}$

26 Ivi: 284 .

27 Claudio Mario Vittore (1977); Flavio Giuseppe (2006); Robert Pullen (1977).

28 Mortella, mirto.

29 Giovane e sottile e rametto di pianta.

30 Cogliere.

31 Emettere umore.

32 Giorgio di Guccio Gucci (1990: 271-272). 


\section{Dcula ${ }^{23}$}

Vol 21, No 23 (July 2020) • DOI: 10.12977/ocula2020-30

Ilaria Sabbatini | «Poma di paradiso». Le descrizioni botaniche nei diari di pellegrinaggio del corpus fiorentino tra XIII $\mathrm{XV}$ Secolo

Se dal punto di vista archeologico e botanico si può identificare il balsamo con la pianta nota come opobalsamus o balsamus, meno semplice è capire se tale pianta fosse presente alla mente dei pellegrini e all'ambiente sociale costituito dai loro lettori. L'opobalsamus è un arbusto del genere Commiphora originario della penisola arabica caratteristico dei climi desertici. Di questo arbusto venivano utilizzate due varietà che erano originarie rispettivamente della regione intorno alla Mecca (Commifora opobalsamus) e dell'Arabia meridionale (Commifora qataf) mentre una terza si era acclimatata sull'altra sponda del Mar Rosso (Commifora abyssina). Di queste solo la prima poté acclimatarsi nella Giudea e le altre due furono oggetto di intensi traffici commerciali. ${ }^{33}$ Le descrizioni dei pellegrini fiorentini non si pongono però su un piano di esattezza tassonomica ma al contrario danno alla pianta del balsamo una significazione religiosa che è collegata con l'idea dell'origine e della destinazione dell'uomo: il paradiso terrestre e il paradiso escatologico si fondono, in questi racconti, passando attraverso la mediazione operata dal Cristo nella resurrezione. In un simile affresco, così carico di risonanze scritturali del Vecchio e del Nuovo testamento, l'elemento cardine intorno a cui tutto ruota risulta essere il potere taumaturgico di Gesù. Da un punto di vista pratico, per i pellegrini fiorentini sussisteva un effettivo problema di identificazione delle varietà vegetali. Per questo motivo le rappresentazioni come quella di Frescobaldi cercano di ovviare al problema cogliendo le caratteristiche oggettive della pianta per descriverla ai propri lettori nel modo più chiaro possibile:

Questo fattore ci menò a vedere il giardino e come si coglie il balsimo, il quale si coglie in questo modo: che levano di quelle foglie che sono intorno al gambo come di basilico, e di quindi esce certe gocciuole bianche a modo di lattificio di fico, e con un poco di bambagia ricolgono questo liquore; e quando hanno inzuppata la bambagia, la premono colle dita in una ampoluzza, e penasi un gran pezzo ad averne un poco. ${ }^{34}$

L'ebreo Mešullam da Volterra spiega le proprietà antisettiche del balsamo. La descrizione del viaggio contenuta nel suo diario è concentrata sui luoghi di culto ebraici e sulle comunità che incontra lungo il percorso: il racconto che questo autore fa dell'oasi di Matarea ha una motivazione diversa dagli autori cristiani. Quello che muove il suo interesse non è tanto l'aspetto simbolico connesso alla leggenda cristiana, di cui comunque riferisce gli elementi salienti, quanto l'efficacia curativa della pianta:

Giuro sulla mia vita di aver visto un poco di quel balsamo nella casa del gran turcimanno, che gli era stato dato dal soldano. Un suo amico, chiamato Muhammad, si era ferito l'alluce del piede sinistro con un'ascia mentre stava tagliando un albero: misero il balsamo su quella ferita, e in tre giorni guarì, e non rimase alcun segno: non ho mai

33 Gnoli (1997).

34 Lionardo Frescobaldi (1991: 150). 


\section{Pcula ${ }^{23}$}

Vol 21, No 23 (July 2020) • DOI: 10.12977/ocula2020-30

Ilaria Sabbatini | «Poma di paradiso». Le descrizioni botaniche nei diari di pellegrinaggio del corpus fiorentino tra XIII $\mathrm{e}$ XV secolo

visto in vita mia un prodigio grande come questo. Quest'olio è scuro e assomiglia all'olio di ricino. 35

Marco di Bartolomeo Rustici, sempre in equilibrio tra descrizione naturalistica e coloritura moralizzata delle sue rappresentazioni, riferisce che il balsamo non si può trapiantare perché le uniche piante che riescono a crescere dando il succo curativo sono quelle che fece nascere il Cristo stesso dalle gocce dei suoi abiti lavati: «Qualunque persona volessi delle dette piante bisongna che vada in que-luogo per esse; e sapiate che molte persone vanno per le piante, pongo-le quivi d'apresso e quelle creschono e non fanno frutto se none quelle che piantò Ihesù Cristo per sua santixima grazia». ${ }^{36}$

Non sappiamo se queste descrizioni siano realistiche, non sappiamo se i pellegrini fiorentini abbiano visto veramente ciò che descrivono, non sappiano se ciò che chiamano balsamo sia effettivamente identificabile con l'opobalsamums individuato dall'archeologia e dalla botanica. Non sarà in questa sede che si potrà dirimere la questione, ma alla fine ciò che conta agli occhi dei viaggiatori non è tanto la corretta classificazione delle specie quanto l'eccezionalità di ciò che vedono e la possibilità di darne testimonianza.

Quello che raccontano i pellegrini è la persistenza del miracolo, tangibile anche quando vedono il balsamo davanti ai loro occhi. La pianta con le sue qualità medicamentose diventa oggetto miracoloso essa stessa, prova concreta e strumento di Grazia efficace per tutti coloro che vi entrano in contatto. Il confine tra effetto medicamentoso e potere taumaturgico si fa labile poiché, nei racconti dei pellegrini, le proprietà del balsamo non derivano da una sua caratteristica naturale bensì dal contatto con il corpo del Salvatore, mediato dall'acqua che è colata dai suoi panni. La pianta non solo reca la memoria viva della presenza del sacro ma perpetra la forza salvifica che l'ha impregnata dal momento in cui è venuta in contatto con la persona divina. Per questa ragione non si tratta solo di una devozione memoriale ma della possibilità effettiva e attuale di attingere alla proprietà salvifica trattenuta dal balsamo. Questa proprietà è concreta, fisica e sensibile come riferisce Gregorio di Tours († 594) a proposito delle reliquie da contatto. Se il devoto desidera prendere una reliquia, spiega infatti il vescovo francese, deve appendere la stoffa all'interno della tomba del santo e una volta rimossa la troverà più pesante per essersi riempita della Grazia divina. ${ }^{37}$

Una analoga concezione della permanenza del prodigio si riscontra nella descrizione delle tracce fisiche lasciate da particolari eventi della vita terrena del Cristo. È il caso della grotta del latte dove, secondo la leggenda, il latte caduto dal seno di Maria ha reso bianca la terra e l'ha dotata di particolari virtù curative. Ciò vale per tutti quei luoghi in cui i resti di miracoli ed eventi danno consistenza materica alle devozioni religiose. Tra questi un ruolo del tutto particolare è riservato al balsamo che con la sua presenza continua a rappresenta-

35 Mešullam da Volterra (1989: 57-58).

36 Marco di Bartolomeo Rustici (2015: 222).

37 Gregorio di Tours (1885: 54). 


\section{Ocula ${ }^{23}$}

Vol 21, No 23 (July 2020) • DOI: 10.12977/ocula2020-30

Ilaria Sabbatini | «Poma di paradiso». Le descrizioni botaniche nei diari di pellegrinaggio del corpus fiorentino tra XIII $\mathrm{XV}$ secolo

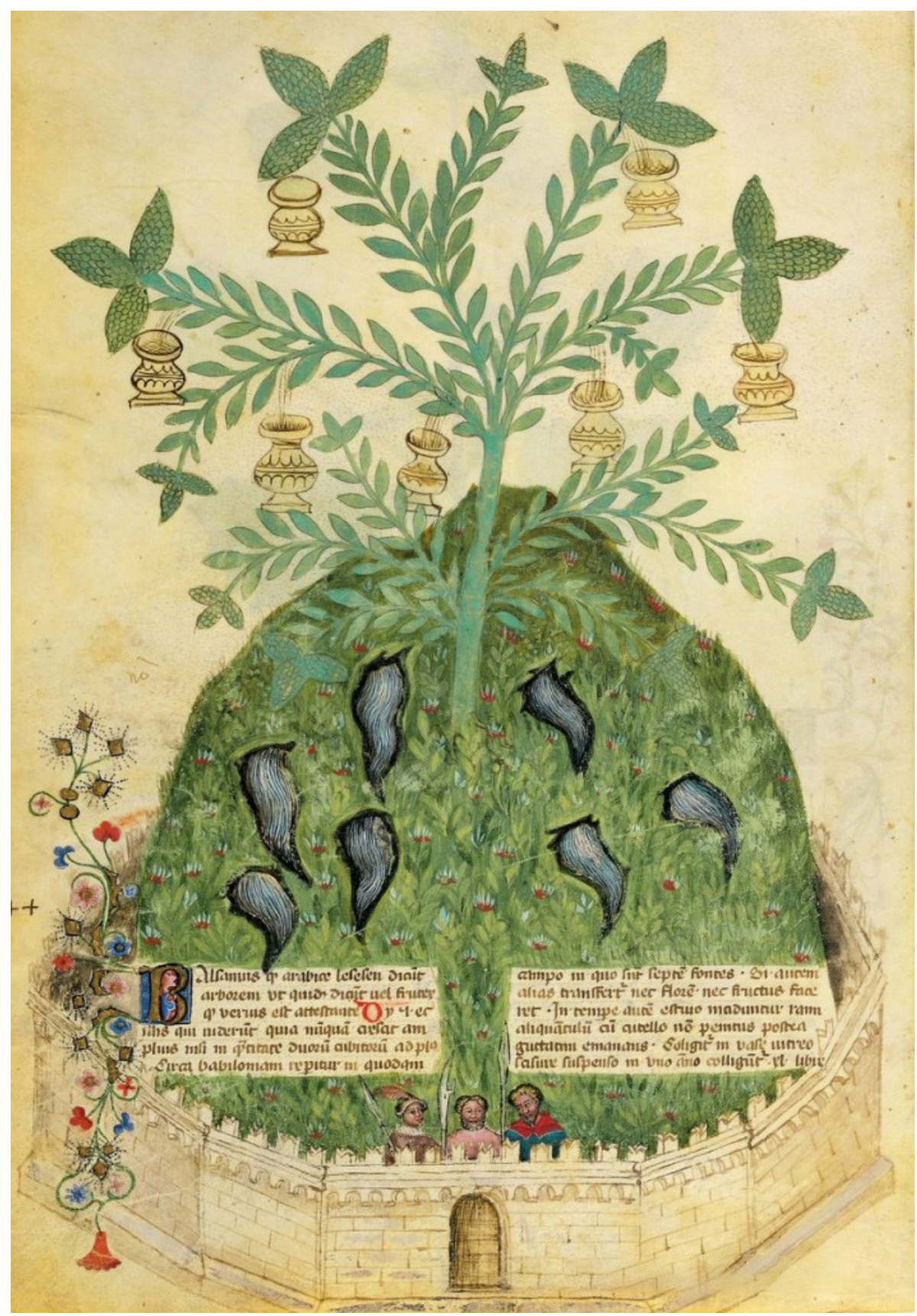

Figura 1. Balsamo. Historia Plantarum, Roma, Biblioteca Casanatense, Ms. 459, xv sec. ex., c. 33v.

re la prova dell'intervento miracoloso, con il suo potere curativo testimonia la forza taumaturgica del Cristo e con l'impossibilità del trasferimento conferma la sua eccezionalità rispetto alle comuni piante che i viaggiatori incontrano nelle loro cerche. 


\section{Dcula ${ }^{23}$}

Vol 21, No 23 (July 2020) • DOI: 10.12977/ocula2020-30

Ilaria Sabbatini | «Poma di paradiso». Le descrizioni botaniche nei diari di pellegrinaggio del corpus fiorentino tra XIII $\mathrm{eXV}$ secolo

\section{La banana paradisiaca}

Oltre al balsamo, il banano è un'altra pianta che attira particolarmente l'attenzione dei pellegrini per il suo frutto straordinario che reca in sé il segno della croce di Cristo. Come si è visto, la narrazione della natura nei diari di pellegrinaggio ha sempre una forte valenza metaforica ma non risponde esclusivamente a logiche simboliche. Se da un lato la memoria del simbolo è compartecipe delle descrizioni della natura, la loro rappresentazione deve fare i conti con la realtà materiale quale si presenta ai sensi dell'osservatore. I pellegrini non si limitano a riportare pedissequamente ciò che hanno sentito o letto da altri ma si impegnano nel trasferire al lettore ciò di cui hanno fatto esperienza in prima persona. È il caso delle muse, ${ }^{8}$ le banane, capaci di solleticare la fantasia, oltre che il gusto, di tutti i viaggiatori dal momento che vengono interpretate come il frutto proibito del paradiso terrestre. Quando però i diaristi fiorentini inseriscono nei loro resoconti le descrizioni delle specie esotiche si trovano a misurarsi con la difficoltà di presentarle a dei lettori per cui tali specie sono spesso sconosciute.

Ecco dunque che le descrizioni vengono costruite facendo ricorso a elementi noti di piante già conosciute che sono giustapposti per illustrare le singole parti. Questa modalità narrativa genera una continua tensione nel testo che permette così di coinvolgere il lettore alla scoperta del nuovo senza dover abbandonare il radicamento nelle proprie conoscenze. Esse diventano anzi la base per sviluppare una comprensione del mondo dell'Oriente mediterraneo anche nel caso particolare delle rappresentazioni vegetali. La banana, secondo il procedimento comparativo sopra esposto, è descritta con un aspetto simile al cetriolo, ma di un sapore talmente dolce da risultare migliore di qualsiasi altro frutto conosciuto. Di conseguenza, i pellegrini fiorentini come Niccolò da Poggibonsi, nel raccontare di tale delizia la identificano senza indugio nel frutto del paradiso terrestre:

Le poma di Paradiso sono fatte così che lo pedale dell'arbore si diventa grosso molto e le foglie sue si ha a modo di lingua; ed è sì grande la detta foglia che parecchi uomini ci giacerebbono su una foglia, e la foglia si è lunga come una lancia ed è larga parecchie braccia. $\mathrm{E}$ in cima del detto arbore si fa uno ramicello con uno fiore, e questo fiore si è fatto come una pigna di pino, ma è troppo più lungo, cioè parecchie spanne; e in tutto l'albero none nasce più che una pannocchia. E le dette poma sono fatte come baccelli; $\mathrm{e}$ quando sono mature, sì le truovi gialle; e quando si cogliono le poma, sì si taglia lo pedale dalle barbe, e poi da indi a xx dì si nasce l'altro pedale; e come è grande, subito fa le poma. Ed è tanto dolce e saporito e soave a mangiare quanto nulla cosa più si possa dire. ${ }^{39}$

I conquistatori musulmani giunti fino all'India importarono le banane in Palestina, successivamente i mercanti arabi le diffusero in quasi tutta l'Africa: il Corano parla già della tanto apprezzata pianta. La cinquantaseiesima sura

38 Così chiamate come adattamento dell'arabo mūza.

39 Niccolò da Poggibonsi (1990: 145-146). 


\section{Dcula ${ }^{23}$}

Vol 21, No 23 (July 2020) • DOI: 10.12977/ocula2020-30

Ilaria Sabbatini | «Poma di paradiso». Le descrizioni botaniche nei diari di pellegrinaggio del corpus fiorentino tra XIII $\mathrm{XV}$ Secolo

contiene una rappresentazione dettagliata del giardino di delizie allestito per i meritevoli e il banano trova la sua centralità proprio nell'eden musulmano, offrendo riparo e ristoro con l'abbondanza dei propri frutti. $4^{40}$ Ancora nel '700, per descrivere la tradizione letteraria relativa alla specie, Linneo faceva riferimento alla credenza secondo cui la banana era il frutto proibito mangiato da Adamo ed Eva. Lo studioso asseriva che questa identificazione era stata sostenuta dal «peregrinatorum vulgus» e poteva trovare giustificazione nella bontà del frutto e nel fatto che una volta tagliato trasversalmente rivela il disegno di una croce. ${ }^{41}$ Il fiorentino Frescobaldi tenta una descrizione tassonomica della pianta basandosi sulle scarse conoscenze botaniche che dovevano essere comuni alla maggior parte degli uomini del suo tempo che non praticassero la scienza medica o la farmacopea: «Le sue foglie sono come d'ella, ${ }^{42}$ ma più lunghe, e 'l suo gambo è come di finocchio, ma è molto più grosso, e seccasi e rimette ogni anno una volta». ${ }^{43}$ Simone Sigoli, come molti altri, si sofferma invece sull'aspetto esterno, sul sapore e infine sul fenomeno della croce visibile in qualsiasi sezione trasversale per via della struttura interna del frutto ma che l'autore interpreta come miracolo:

Ancora v'è un frutto si chiama muse del quale dicono peccò Adamo, ed è fatto come cittriuoli ma sono più lunghi et più sottili et più dilicati. Et sono molto teneri et d'estratto di sapore dalle nostre cose, per modo che chi ll'usasse ne lascierebbe ogni altra cosa. In questo fructo si vede un gran miracolo: quando tu 'l parti, inn ogni modo si parte si vede il Crocifisso. Et di ciò facemo pruova, et per molti si chiama "pome di paradiso". L'albero del detto fructo è di colore sanguigno ed è sollo et dilicato, non troppo grosso et va alto dalle III in V braccia. Et le fogle sue sono come lella nostra di qua, salvo quelle sono lunge bene inI braccia. Et questo così facto pedale fa una volta fructo et non mai più. Et poi si secca rimette da piè un altro ghambo per lo simile, facciendo una volta frutto et non più. ${ }^{44}$

Come si può facilmente capire la conoscenza botanica di questi autori non è particolarmente solida ed ecco allora che mettono in atto una strategia alternativa: si impegnano a costruire la descrizione dell'oggetto sconosciuto per approssimazione attraverso l'accostamento alle cose conosciute. Le osservazioni sul campo si mescolano con le conoscenze pregresse, i dati raccolti per esperienza diretta si intrecciano col repertorio tradizionale delle leggende producendo un'unione inscindibile. La descrizione della natura e la sua semantizzazione religiosa non sono affatto in antitesi nella percezione dei viaggiatori medievali, risulterebbe anzi errata la riduzione della dimensione miracolistica alla categoria dell’irreale. Ciò che riguardava le proprietà delle piante coesisteva senza confliggere con il significato spirituale che veniva

40 Corano, LXVI, 27-32.

41 Linnaeus (1736: 43-44).

42 Edera.

43 Lionardo Frescobaldi (1991: 137).

44 Simone Sigoli (1999: 71-72). 


\section{Ocula ${ }^{23}$}

Vol 21, No 23 (July 2020) • DOI: 10.12977/ocula2020-30

Ilaria Sabbatini | «Poma di paradiso». Le descrizioni botaniche nei diari di pellegrinaggio del corpus fiorentino tra XIII $\mathrm{XV}$ secolo

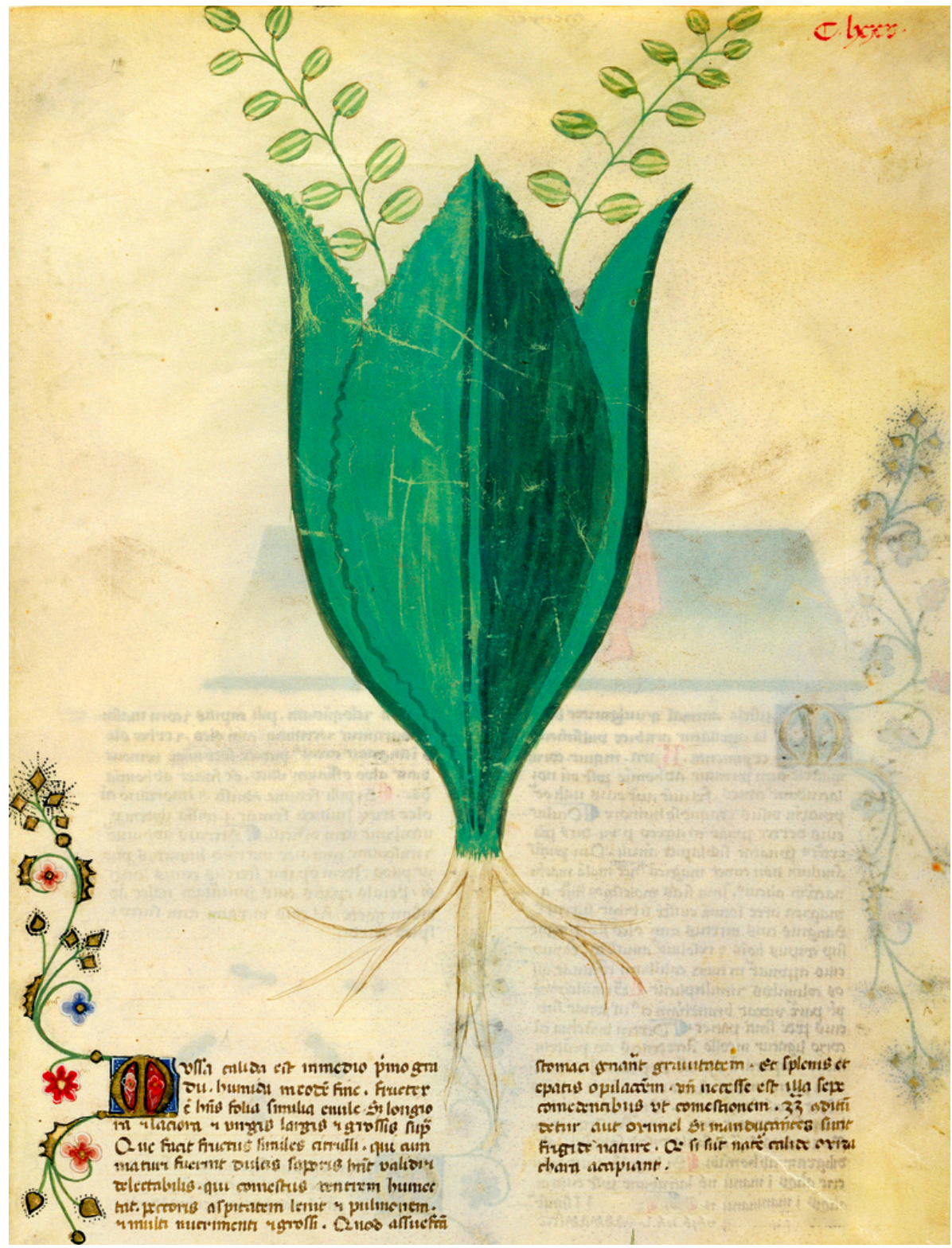

Figura 2. Banano, Historia Plantarum, Roma, Biblioteca Casanatense, Ms.459, xv sec. ex., c. 165v.

loro attribuito. L'immaginario religioso e la descrizione floreale trovavano il loro punto di raccordo in un approccio narrativo che aveva la sua ragione d'essere nella contiguità con la scrittura pratica dei libri di famiglia propri del clima culturale e dell'ambiente mercantile in cui erano immersi gli autori.

\section{Conclusioni}

Nell'ambito della società fiorentina medievale l'interrelazione reciproca tra le classi sociali aveva dato luogo a una contaminazione assai accentuata sul piano della mentalità, dell'etica condivisa e dell'interpretazione stessa del 


\section{Ocula ${ }^{23}$}

Vol 21, No 23 (July 2020) • DOI: 10.12977/ocula2020-30

Ilaria Sabbatini | «Poma di paradiso». Le descrizioni botaniche nei diari di pellegrinaggio del corpus fiorentino tra XIII $\mathrm{e} X \mathrm{~V}$ secolo

mondo. Tutte le funzioni sociali e religiose convergevano sulle grandi famiglie non meno delle responsabilità economiche e politiche. Questa situazione ingenerava una comunanza di sistemi culturali e modelli di pensiero che finivano per diventare collettivi. Firenze era la più grande città mercantile dell'Italia comunale, il commercio costituiva il fondamento su cui il ceto borghese prosperava e i viaggi in ambienti nuovi erano considerati un'eccellente occasione di apprendimento..$^{45}$ Attraverso l'esperienza del pellegrinaggio non si compiva semplicemente un gesto fuori dall'ordinario ma si affermava anche l'adesione a un sistema valoriale condiviso grazie alla doppia valenza del viaggio come pratica religiosa pubblica e come occasione di conoscenza del mondo esterno. Di conseguenza la narrazione del pellegrinaggio risultava impregnata di pragmatismo secolare non meno che di afflato religioso. Il significato del viaggio, che nell'ambito dell'etica mercantile rivestiva una particolare importanza tecnica e pratica, andava coniugandosi con un altro ordine di valori completamente afferente all'ambito spirituale. Tale ambivalenza si manifestava nella descrizione degli ambienti con cui i pellegrini entravano in contatto. L'attenzione per i dati naturalistici che venivano rappresentati nella loro concretezza coesisteva con l'importanza conferita ai luoghi della memoria storica di Cristo, dove erano ancora visibili i segni della manifestazione del divino. La descrizione naturalistica dei diari di pellegrinaggio lasciava sempre spazio a una significazione simbolica letteralmente impregnata di memorie devote. Queste tradizioni strutturavano la rappresentazione del sacro in tutti i racconti dei Luoghi Santi: la loro presenza scandiva le descrizioni della natura, dell'oasi e della sorgente, del balsamo e del banano facendole diventare parte integrante di un vero e proprio circuito del sacro. ${ }^{46}$

\section{Bibliografia}

Ashtor, Eliyahu

1982 Storia economica e sociale del Vicino Oriente nel Medioevo, Torino, Einaudi.

Bartolini, Gabriella; Cardini, Franco (a cura di)

1991 «Nel nome di Dio facemmo vela». Viaggio in Oriente di un pellegrino medievale, Bari, Laterza.

Branca, Vittore (a cura di)

1986 Mercanti scrittori. Ricordi nella Firenze tra Medioevo e Rinascimento, Milano, Rusconi.

Cardini, Franco

2002 In Terrasanta. Pellegrini italiani tra Medioevo e prima età moderna, Bologna, Il Mulino.

Cardini, Franco (a cura di)

1982 Toscana e Terrasanta nel medioevo, Firenze, Alinea.

45 Patrone (1974: 324); Pezzarossa (1990); Mohlo (2000); Gazzini (2002); Nanni (2010).

46 Sabbatini (2018). 


\section{Ocula ${ }^{23}$}

Vol 21, No 23 (July 2020) • DOI: 10.12977/ocula2020-30

Ilaria Sabbatini | «Poma di paradiso». Le descrizioni botaniche nei diari di pellegrinaggio del corpus fiorentino tra XIII $\mathrm{e}$ XV secolo

Cardini, Franco; Miglio, Massimo

2002 Nostalgia del Paradiso. Il giardino medievale, Bari, Laterza.

Chélini, Jean; Branthomme, Henry

2004-6 Le vie di Dio. Storia dei pellegrinaggi cristiani dalle origini al Medioevo, 2 voll., Milano, Jaca Book.

Cicchetti, Angelo; Mordenti, Raul

1982 "La scrittura dei libri di famiglia", in Asor Rosa, A. (a cura di), Letteratura italiana, vol. III, Le forme del testo, II, La prosa, Torino, Einaudi, pp. 1117-1159.

1985 I libri di famiglia in Italia, vol. I, Filologia e storiografia letteraria, Roma, Edizioni di Storia e Letteratura.

Claudio Mario Vittore

1977 Commentariorum in Genesim libri tres, in Migne, J.P. (a cura di), Patrologiae cursus completus. Series Latina, v. Lxi, coll. 937-970, Turnhout, Brepols.

Craveri, Marcello (a cura di)

2002 I vangeli apocrifi, Torino, Einaudi.

Flavio, Giuseppe

2006 Antichità giudaiche, a cura di Moraldi, L., Torino, Utet.

Frescobaldi, Lionardo

1991 Viaggio in Egitto e in Terra Santa, in Bartolini, G. e Cardini, F. (a cura di) «Nel nome di Dio facemmo vela». Viaggio in Oriente di un pellegrino medievale, Bari-Roma, Laterza, pp. 124-196.

Frugoni, Chiara

2008 "La rappresentazione del paesaggio nel Medioevo", in Mencaroni Zoppetti, M. (a cura di), D’erbe e piante adorno. Per una storia dei giardini a Bergamo, percorsi tra paesaggio e territorio, Bergamo, Ateneo di Scienze Lettere e Arti di Bergamo, pp. 33-53.

Fumagalli, Vito

2006 Paesaggi della paura. Vita e natura nel medioevo, Bologna, Il Mulino.

Gazzini, Marina

2002 Dare et habere. Il mondo di un mercante milanese del Quattrocento, Firenze, Firenze University Press.

Gnoli, Tommaso

1997 "La produzione del balsamo nell'oasi di Engaddi (Israele). Su alcuni nuovi documenti dal deserto di Giuda”, in Avanzini A. e Salmeri G. (a cura di), Profumi d'Arabia. Atti del convegno, Roma, «L'Erma» di Bretscheneider, pp. 413-430.

Graboïs, Aryeh

1998 Le pèlerin occidental en Terre sainte au moyen âge, Parigi, De Boeck Université.

Gregorio di Tours

1885 Liber in Gloria Martyrum, in Monumenta Germaniae Historica. Scriptores rerum merovingicarum, I, Hannover, pp. 34-431. 


\section{Ocula ${ }^{23}$}

Vol 21, No 23 (July 2020) • DOI: 10.12977/ocula2020-30

Ilaria Sabbatini | «Poma di paradiso». Le descrizioni botaniche nei diari di pellegrinaggio del corpus fiorentino tra XIII e XV secolo

Gucci, Giorgio di Guccio

1990 Viaggio ai Luoghi Santi, in Lanza, A. e Troncarelli, M. (a cura di), Pellegrini scrittori. Viaggiatori toscani del Trecento in Terrasanta, Firenze, Ponte alle Grazie, pp. 271-438.

Linnaeus, Carl

1736 Musa cliffortiana florens Hartecampi, Haarlem.

Marco di Bartolomeo Rustici

2015 Dimostrazione dell'andata e viaggio al Santo Sepolcro e al Monte Sinai, a cura di Olive, K. e Newbigin, N., in AA.VV., Codice Rustici, vol. II, Firenze, Olschki, pp. 103-295.

Mešullam da Volterra

1989 Il viaggio in terra d'Israele, a cura di Veronese, A., Rimini, Luisè.

Michele da Figline

2010 Viaggio, in M. Montesano, Da Figline a Gerusalemme. Viaggio del prete Michele in Egitto e in Terrasanta (1489-149o), Roma, Viella, pp. 46-166.

Mohlo, Anthony

2000 "Names, Memory, Public Identity in Late Medieval Florence”, in Ciappelli, G. e Lee Rubin, P. (a cura di), Art, Memory, and Family in Renaissance Florence, Cambridge, Cambridge University Press, pp. 237-252.

Mordenti, Raul

1997 I libri di famiglia in Italia, vol. II, Geografia e storia, Roma, Edizioni di Storia e Letteratura.

Nanni, Paolo

2010 Ragionare tra mercanti. Per una rilettura della personalità di Francesco di Marco Datini (1335ca-1410), Pisa, Pacini.

2017 "Per un quadro ambientale e biologico. Il periodo caldo medievale e la variabilità climatica”, in La crescita economica dell'Occidente medievale. Un tema storico non ancora esaurito, Atti del Convegno Internazionale del Centro Italiano di Studi di Storia e Arte (Pistoia, 14-17 maggio 2015), Roma, Viella, pp. 69-91.

Niccolò da Poggibonsi

1990 Libro d'Oltremare, in Lanza, A. e Troncarelli, M. (a cura di), Pellegrini scrittori. Viaggiatori toscani del Trecento in Terrasanta, Firenze, Ponte alle Grazie, pp. 31-158.

Oursel, Raimond

1988 Pellegrini del medioevo, gli uomini le strade, $i$ santuari, Milano, Jaca Book.

1993 Vie di pellegrinaggio e santuari da Gerusalemme a Fatima, Milano, Jaca Book.

Patrone, Anna Maria

1974 L'ascesa della borghesia nell'Italia comunale, Torino, Loescher.

Pezzarossa, Fulvio

1990 "Una prima verifica dei rapporti fra strumenti culturali e ruoli sociali: la memorialistica e i ceti bolognesi nei secoli XIV-XVII", in Sapere e/è potere. Discipline, dispute e professioni nell'Università Medievale e Moderna. Il caso 


\section{Ocula ${ }^{23}$}

Vol 21, No 23 (July 2020) • DOI: 10.12977/ocula2020-30

Ilaria Sabbatini | «Poma di paradiso». Le descrizioni botaniche nei diari di pellegrinaggio del corpus fiorentino tra XIII $\mathrm{e} X \mathrm{~V}$ secolo

bolognese a confronto, Atti del IV Convegno (Bologna 13-15 aprile 1989), vol. III, Dalle discipline ai ruoli sociali, Bologna, Istituto per la storia di Bologna, pp. 111-134.

Rao, Riccardo

2015 I paesaggi dell'Italia medievale, Roma, Carocci editore.

Richard, Jean

1981 Les récits de voyages et de pèlerinages, «Typologie des sources du Moyen Age occidental», vol. 30, Turnhout, Brepols.

Robert Pullen

1977 Sententiarum libri octo, in Migne, J.P. (a cura di), Patrologiae cursus completus. Series Latina, v. CLXXXVI, coll. 639-1010, Turnhout, Brepols.

Sabbatini, Ilaria

2012 “"Finis corporis initium animae». La qualità morale del nemico nella rappresentazione del corpo. Un excursus tra patristica, epica crociata e odeporica di pellegrinaggio", Micrologus, XX, SISMEL, Firenze, pp. 257-292.

2014 " "Com’io cercai di molti luoghi santi». Il corpus fiorentino dei diari di pellegrinaggio a Gerusalemme", in Musarra, A. (a cura di), Gli Italiani e la Terrasanta, SISMEL-Edizioni Del Galluzzo, Firenze, pp. 123-134.

2015 "«Tutti erano ignudi e tutti neri». La fisiognomica dell'alterità musulmana", in Deformità Fisica e Identità della persona tra medioevo ed età moderna, Atti del XIV Convegno internazionale di studi del Centro Studi sulla Civiltà del Tardo Medioevo di San Miniato (San Miniato, 21-23 settembre 2012), Firenze, Firenze University Press, pp. 355 - 389.

2018 "Il modello della civitas e la descrizione dello spazio sacro nei pellegrini scrittori di Terra Santa", in AA. VV., La città, il viaggio, il turismo Percezione, produzione e trasformazione, vII Congresso AISU, Napoli, 7-8-9 settembre 2017, Napoli, CIRICE Edizioni, pp. 293 - 302.

2019 "Itineraria, descriptiones e diari di pellegrinaggio. Il libro quinto del Codex Calixtinus all'incrocio dei generi”, in Sabba, F. (a cura di), Il patrimonio culturale attraverso le esperienze di viaggio. Prima e dopo il Gran Tour, Napoli, Associazione culturale Viaggiatori - Dipartimento di Beni Culturali, Università degli Studi di Bologna, pp. 17-31.

Scafi, Alessandro

2006 Mapping Paradise. A History of Heaven on Earth, London, British Library.

2019 "Natura perfetta nell'Eden. Un'utopia medievale”, in Catapano, G. e Grassi, O. (a cura di), Rappresentazioni della natura nel Medioevo, Firenze, SISMELEdizioni Del Galluzzo, pp. 3-25.

Sigoli, Simone

1999 Mentione delle terre d'oltre mare, in Bedini, A., Testimone a Gerusalemme. Il pellegrinaggio di un fiorentino nel Trecento, Roma, Città Nova, pp. 69-138.

Sumption, Jonathan

1993 Monaci santuari e pellegrini, Roma, Editori Riuniti.

Todeschini, Giacomo

2007 Visibilmente crudeli. Malviventi, persone sospette e gente qualunque dal medioevo all'età moderna, Bologna, il Mulino. 


\section{Dcula ${ }^{23}$}

Vol 21, No 23 (July 2020) • DOI: 10.12977/ocula2020-30

Ilaria Sabbatini | «Poma di paradiso». Le descrizioni botaniche nei diari di pellegrinaggio del corpus fiorentino tra XIII e XV secolo

Ilaria Sabbatini è Research fellow presso SISMEL (Società Internazionale per lo Studio del Medioevo Latino) per cui è responsabile del progetto ARVO - Archivio Digitale del Volto Santo. È ideatrice dello Young Historians Festival di Lucca in convenzione con il Centro internazionale di Didattica della Storia e del Patrimonio (DiPaSt) dell'Università degli Studi di Bologna e con il patrocinio del Festival del Medioevo di Gubbio. Sta curando il ciclo di incontri Toscana Matildica in collaborazione con enti locali, regionali e Istituti Storici. È consulente di vari enti pubblici e istituzioni museali. È docente del Master in "Analisi delle Fonti e Metodologia della Ricerca Storica” LUMSA. Svolge attività di insegnamento nel corso: Produzione documentaria nel medioevo e implementazione digitale delle fonti. Fa parte del comitato editoriale della collana Viatores (Maria Pacini Fazzi), della collana A EstOvest (Textus Edizioni), della rivista La porta d'Oriente (Pagine), della rivista Almaturism (Università di Bologna), della collana Matildica (Patròn Editore), della collana Peregrinantes in mundo (Edizioni la Vela). È direttrice della collana editoriale Toscana Matildica (La Villa). Ha all'attivo numerose pubblicazioni scientifiche nonché partecipazioni a convegni nazionali e internazionali. 